\title{
Future Metrology Needs for Synchrotron Radiation Grazing-Incidence Optics
}

\author{
L. Assoufid ${ }^{1}$, O. Hignette ${ }^{2}$, M. Howells ${ }^{3}$, S. Irick ${ }^{3}$, H. Lammert ${ }^{4}$, P. Takacs ${ }^{5}$
}

\author{
'User Program Division, Argonne National Laboratory, 9700 South Cass Avenue, Argonne IL 60439. \\ ${ }^{2}$ ESRF, Avenue des Martyrs, B.P. 220, F-38043, Grenoble Cedex 09, France. \\ ${ }^{3}$ Lawrence Berkeley National Laboratory, 1 Cyclotron Road, Berkeley, CA 94720 , USA. \\ ${ }^{4}$ BESSY GmbH, Albert-Einstein-Straße 15, D-12489 Berlin, Germany. \\ 'Optical Metrology Laboratory, Bldg. 535B, Brookhaven National Laboratory, Upton, NY 11973, USA.
}

\begin{abstract}
An International Workshop on Metrology for X-ray and Neutron Optics, the first of its kind, was held March 16-17, 2000, at the Advanced Photon Source at Argonne National Laboratory. Metrology specialists, beamline engineers and scientists, and vendors from around the world met to evaluate current metrology instrumentation and methods used to characterize the surface figure and finish of long grazing-incidence optics used in synchrotron radiation beamlines, and to consider future needs for synchrotron, FEL, and neutron sources. This paper summarizes the discussions on mirror and metrology requirements for the current and next-generation x-ray sources. Some recommended strategies for the needs of the future are also given.
\end{abstract}

Keywords: Optical metrology, synchrotron radiation, FEL, grazing-incidence mirrors, surface figure, surface finish

\section{Introduction}

Current (third-generation) synchrotron radiation (SR) sources deliver photon beams with high brilliance and moderate coherence. Full exploitation of these features and preservation of brilliance of the $x$-rays has required the use of grazing-incidence mirrors typically $1 \mathrm{~m}$ long or more, characterized by having surface figure errors below 3 $\mu \mathrm{rad}$ rms and surface roughness errors less than $3 \AA$ rms. These requirements, along with others for high heat loading and ultrahigh vacuum-compatible materials, have imposed stringent demands on mirror manufacturers to produce better mirrors. Successful collaborations have resulted between synchrotron radiation beamline scientists and mirror vendors to produce mirrors with sufficient quality to meet the challenges of these third-generation beam lines. Special fabrication tools were created, and a specialized metrology tool, the long trace profiler (LTP), was developed' to measure surface figure and curvature of long aspheres. Standard commercial instruments were adapted to evaluate the figure and finish of these large optics. In particular, the $\mathrm{x}$-ray astronomy community continues to improve the performance of their large spherical mirrors. As an example, NASA's Advanced X-ray Astrophysics Facility (AXAF) has four double-bounce reflectors $80 \mathrm{~cm}$ long and up to $120 \mathrm{~cm}$ diameter, which achieve an overall system resolution of $2.5 \mu$ radian. On the other hand, the ultimate surface accuracy is being achieved by EUV lithography optics as exemplified by the Euclides project (ASML, Zeiss, and Oxford). This group has produced 2.5 $\AA \mathrm{rms}$ and both high- and mid-spatial-frequency roughness below $4 \AA \mathrm{rms}$.

Even with these advances in mirror fabrication technology, residual imperfections on mirrors (as well in other optical x-ray SR components) were found to degrade the quality of $x$-ray beams from third-generation sources, ${ }^{2-3}$ and the techniques for maintaining $\mathrm{x}$-ray beam quality (emittance and coherence) are still evolving. Moreover, $\mathrm{x}$-ray free-electron laser (FEL) sources currently planned around the world, such as the Linac Coherent Light Source (LCLS, at SLAC in Stanford) and the X-ray TESLA FEL (Germany), are expected to deliver $x$-ray beams that are fully coherent and with peak brightness more than ten orders of magnitudes higher than that of the current synchrotron radiation sources. Therefore, tolerances on optical elements matching the photon beam properties will become even tighter. Typical good figure errors obtained currently on SR mirrors are on the order of $1 \mu \mathrm{rad}$ rms on a $1 \mathrm{~m}$ long, depending on the mirror size and shape. Even these tolerances will be inadequate to meet the needs of next-generation sources. This raises new challenges for both mirror polishing and metrology.

The workshop provided excellent opportunities to evaluate current metrology capabilities around the world and to stimulate innovation on future instruments and techniques.

The submitted manuscript has been created: by the University of Chicago as Operator of ; Argonne National Laboratory ("Argonne") under Contract No. W-31-109-ENG-38 with the U.S. Department of Energy. The U.S. Government retains for itself, and others acting on its behalf, a paid-up, nonexclusive, irrevocable worldwide license in said article to reproduce, prepare derivative works, distribute copies to the public, and perform publicly and display publicly, by or on behalf of the Government. 


\section{DISCLAIMER}

This report was prepared as an account of work sponsored by an agency of the United States Government. Neither the United States Government nor any agency thereof, nor any of their employees, make any warranty, express or implied, or assumes any legal liability or responsibility for the accuracy, completeness, or usefulness of any information, apparatus, product, or process disclosed, or represents that its use would not infringe privately owned rights. Reference herein to any specific commercial product, process, or service by trade name, trademark, manufacturer, or otherwise does not necessarily constitute or imply its endorsement, recommendation, or favoring by the United States Government or any agency thereof. The views and opinions of authors expressed herein do not necessarily state or reflect those of the United States Government or any agency thereof. 


\section{DISCLAIMER}

Portions of this document may be illegible in electronic image products. Images are produced from the best available original document. 


\section{General X-ray Mirror Requirements and Issues}

Although the workshop's main focus was metrology instrumentation, various aspects of SR optics, such as fabrication, and material specifications and requirements, were also discussed. The workshop began with a brief review of the history of synchrotron optics and a forward look at optical systems and metrology needs (Malcolm Howells, ALS/LBNL). Howells suggested standardization of optical components as a means to save cost and time in developing new beam lines. Sunil Sinha (APS/ANL) presented the framework for understanding the effects of surface roughness and figure error on mirror performance and coherence preservation. He compared various techniques for evaluating diffuse scatter and speckle, including $\mathrm{x}$-ray bidirectional reflectance distribution function (BRDF), scanning probe microscopes, and optical profilometers.

\subsection{Specifications and standardization}

Figure and finish requirements for SR mirrors are traditionally specified with two single-number parameters: one for rms surface roughness and the other a rms slope error value. However, experience at SR facilities showed that specifying a mirror using these two single values might be inadequate. An upper bound on the power spectral density function (PSD) would be a better choice (Howells). Performance-related specifications were also suggested (Howells). The idea consists of specifying an optic that will deliver light from a source to a slit (say) by requiring that a certain \% of photons must be delivered by the optic in a test with the given source/slit geometry.

\section{Metrology Instrumentation Requirement and Developments}

Standard metrology instruments used to inspect synchrotron radiation optical components are of a non contact type and are typically composed of a long trace profiler (LTP) for measuring surface slope error and curvature, a figure interferometer, and a roughness-measuring instrument. The roughness-measuring instrument is generally an interference visible light microscope, but an atomic microscope can be a very good complementary tool. These instruments, generally housed in a clean room (of Class 10,000 or better) with a well-controlled environment, are chosen to cover the wide range of spatial frequency needed for a complete characterization of an optic.

\subsection{The long trace profiler characterization, development and innovations}

The LTP, as developed by Peter Takacs and Shinan Qian (BNL), 'is so far the only instrument available to the SR community to directly measure with submicroradian accuracy the slope of long, aspherical mirrors used in SR beamlines. Many variations of this instrument have been used around the world. Each LTP is unique in that it is usually upgraded by its owner with customized hardware and measurement techniques for improving accuracy and versatility. The current performance of a commercial standard LTP, the LTP II system, is at the $0.5 \mu \mathrm{rad}$ rms level (Takacs). Optics with a much lower slope error limit $(0.1 \mu \mathrm{rad})$ are now in demand, and there is clearly a need to improve the performance level below this limit. However, sources of errors for the current LTPs were identified, and ways to mitigate them along with performance expectations were presented by Takacs, Steve Irick (LBNL), Heiner Lammert (BESSY), and Giovanni Sostero (ELETTRA). As with all ultraprecision measuring machines, the major error source limiting the LTP performance is environmental instability. Temperature control at the $\pm 0.1^{\circ} \mathrm{C}$ level is adequate for a $0.5 \mu \mathrm{rad}$ rms system noise level, but significant improvement in the thermal control of the local environment will be necessary to get down to the $0.1 \mu \mathrm{rad}$ rms accuracy and repeatability level. The target is an accuracy level on the order of $0.05 \mu \mathrm{rad}$, which can be achieved by stabilizing the temperature to within \pm 0.033 ${ }^{\circ} \mathrm{C}$ (Takacs). The inhomogeneity in the index of refraction of the LTP transmitting optical components (prisms, lenses, etc.) is also a source of error (Heiner Lammert, BESSY II). The error induced by the variation in the index of refraction is particularly important when evaluating curved surfaces with small radii (large test beam deflection angle). Lammert has estimated that the variation in the index of refraction, $\Delta n$, of LTP transmission optical elements should not exceed $10^{-8}$.

Beside the standard version, several new LTP concepts being currently developed were discussed during this workshop by François Polack (LURE), Ajay K. Saxena (Indian Institut of Astrophysics), Ingolf Weingărtner (PTB), and Qian (BNL).

\subsection{Interferometers performance, limitations and developments}

Commercial interferometers are generally not optimized for evaluation of SR mirrors, and their performance has not reached the fundamental limits yet (Chris Evans, NIST). Environment stability is one of the major limiting factors 
along with a traceability issue. Another limitation comes from their reliance on a reference mirror for measurements, and they are only suitable for weak aspheres. Weingartner (PTB) discussed a high-resolution largearea curvature-scanning device that can be used for steep aspheres. He also described a method for extracting the shape from the curvature without cumulative errors. In the past, such errors have prevented this otherwise attractive approach from working correctly with large optics. Raymond Mercier (Institut d'Optique, Orsay) developed a figure interferometer to test XUV optics with a noise level to $0.2 \mathrm{~nm} \mathrm{rms,} \mathrm{also} \mathrm{much} \mathrm{lower} \mathrm{than} \mathrm{for} \mathrm{a} \mathrm{standard}$ interferometer. Alain Dubois (Ecole Superieure de Physique et de Chimie Industrielle de Paris, Paris) discussed a roughness instrument built around a Nomarski polarizing differential microscope. The instrument yields a differential roughness profile instead of a topographic profile but requires no reference mirror and has a very low noise level of $5 \mathrm{pm}$ compared to commercial instruments. Standard figure interferometers have a typical aperture of about $150 \mathrm{~mm}$ diameter. Therefore, measurements of long grazing-incidence mirrors have to be performed at an angle. Michael Bray (MB Optique) proposed stitching interferometry as an alternative to overcome the aperture limitation. The idea is to use a conventional figure interferometer to make a sufficient number of overlapped submeasurements to cover the entire mirror surface. Then these measurements are stitched together numerically to compose a complete 3-D mirror surface. The algorithm used was impressively successful in the view of many unsuccessful attempts at the same problem in the past.

\subsection{X-ray in situ metrology}

Because evaluation can be done with radiation of the relevant wavelength and within the natural environment of an experiment, synchrotron radiation is obviously the ultimate tool for testing and characterizing an optic. However, because in situ metrology requires rather complicated and expensive equipment, it can only be used as a complementary method, not as a tool for inspection of delivered optics. Olivier Hignette (ESRF) used x-ray long trace profilometry to measure mirror slope errors with 50 nrad accuracy, much below the current accuracy of the current LTPs. He also described beamline wavefront analysis and optimization techniques as a means to adjust and tune either individual components or a beam line as whole.

In situ metrology is a valuable tool for monitoring the long term stability of a beam line optical system. Therefore, it needs to be installed permanently and be quickly deployable.

\section{FEL Sources}

Looking ahead, FEL light sources proposed around the world are expected to deliver photon beams with unprecedented properties. Because of the lack of experience with FELs operating in the x-ray regime, no precise quantities for surface figure or finish requirements were presented for beam line optics in these machines. However, we are aware that FEL sources will be characterized by peak brightness ten orders of magnitude higher than the current third-generation sources. Moreover, FELs will deliver a photon beam that is fully coherent, with a transverse coherence length much larger than that of the current third-generation synchrotron sources. Therefore, subnanometer roughness levels now achieved over millimeter length scales must now be extended over longer lateral scale lengths in order to preserve the intrinsic beam coherence (Sinha, APS/ANL). These tighter tolerances along with the mirror material requirements provide a challenge to both mirror manufacturers and metrologists. However, promising techniques to prepare a new generation of mirror are being developed. For example, the ion beam figuring technique has been described as a way to further improve conventionally polished substrates. A $300 \mathrm{~mm} \mathrm{Si}$ substrate prepared using this method (by ESRF and ZEISS) yielded a surface slope error of $0.1 \mu \mathrm{rad} r \mathrm{rs}$.

\section{Conclusions}

The optical quality of grazing-incidence mirrors has dramatically improved over the last decade. However, further improvement in mirror quality is necessary in order to meet future demand from both the current- and nextgeneration synchrotron light sources. The current interferometer-based instruments have not yet reached the fundamental limits. Environment stability is one of the major limiting factors. The performance of the current LTP can be further improved beyond the existing limit $(0.5 \mu \mathrm{rad})$ by excellent temperature stability and better optomechanical components. The target is an accuracy level on the order of $0.05 \mu \mathrm{rad}$, which can be achieved by stabilizing the temperature to within $\pm 0.033{ }^{\circ} \mathrm{C}$. Standards in metrology methods are highly desirable, and standards in optical components would save cost and time. A standard wave-optics simulation code for designing and predicting the performance of optics and beamlines is needed. Such predictions based on optical metrology measurements also need to be implemented. The workshop was successful in addressing the problems faced by metrologists, beamline scientists and engineers, and vendors. A follow-up meeting was suggested, and ESRF was 
the proposed host in two years time.

\section{Acknowledgments}

L. Assoufid would like to thank D. Mills and A. Macrander for their support. He also would like to acknowledge the staff of ANL Conference Services and APS Users Office for their help in the workshop organization. This work is supported by the U.S. Department of Energy, BES-Materials Sciences, under contracts W-31-109-ENG-38, DE-AC02-98CH10886, and DC-AC03-76SF00098.

\section{References}

1. P.Z. Takacs, and S. Qian, United States Patent 4884697 (1989).

2. Snigirev et al., Nucl. Instrum. Methods A370, pp. 634-640, 1996.

3. G. Srajer and J. Lang, Argonne National Laboratory (unplished), 1997.

4. S.C. Irick, "Long Trace Profiler Survey Results," Proc. SPIE, Vol. 3782, (1999) p. 5. 